\title{
Intratubular amyloid in light chain cast nephropathy is a risk factor for systemic light chain amyloidosis
}

Jean-Baptiste Gibier ${ }^{1}$, Viviane Gnemmi ${ }^{1}$, François Glowacki ${ }^{2}$, Eileen M Boyle ${ }^{3}$, Benjamin Lopez ${ }^{4}$, Evelyne MacNamara ${ }^{5}$, Maxime Hoffmann $^{6}$, Raymond Azar ${ }^{7}$, Thomas Guincestre ${ }^{8}$, Franck Bourdon ${ }^{9}$, Marie-Christine Copin ${ }^{1}$ and David Buob ${ }^{10}$

${ }^{1}$ University of Lille, Department of Pathology, Centre de Biologie Pathologie, CHU Lille, Lille, France;

${ }^{2}$ University of Lille, Department of Nephrology, Hôpital Claude Huriez, CHU Lille, Lille, France; ${ }^{3}$ University of Lille, Department of Hematology, Hôpital Claude Huriez, CHU Lille, Lille, France; ${ }^{4}$ University of Lille, Department of Immunology, Centre de Biologie Pathologie, CHU Lille, Lille, France; ${ }^{5}$ Department of Nephrology, Centre Hospitalier de Béthune-Beuvry, Béthune, France; ${ }^{6}$ Department of Nephrology, Hôpital Privé de La Louvière, Lille, France; ${ }^{7}$ Department of Nephrology, Centre Hospitalier de Dunkerque, Dunkerque, France; ${ }^{8}$ Department of Nephrology, Centre Hospitalier de Roubaix, Roubaix, France; ${ }^{9}$ Department of Nephrology, Clinique du Bois, Lille, France and ${ }^{10}$ AP-HP, Hôpital Tenon, Department of Pathology, Paris, France

Light chain cast nephropathy is the most common form of kidney disease in patients with multiple myeloma. Light chain casts may occasionally show amyloid staining properties, that is, green birefringence after Congo red staining. The frequency and clinical significance of this intratubular amyloid are poorly understood. Here, we retrospectively assessed the clinicopathological features of 60 patients with histologically proven light chain cast nephropathy with a specific emphasis on intratubular amyloid, especially, its association with extrarenal systemic light chain amyloidosis. We found intratubular amyloid in 17 cases $(17 / 60,28 \%)$ and it was more frequent in patients with $\lambda$ light chain gammopathy (13/17 in the 'intratubular amyloid' group vs 19/43 in the 'no intratubular amyloid' group, $P=0.02)$. Pathological examination of extrarenal specimens showed that intratubular amyloid was significantly associated with the occurrence of systemic light chain amyloidosis $(5 / 13$ in the 'intratubular amyloid' group vs $0 / 30$ in the 'no intratubular amyloid' group, $P=0.001$ ). Our results indicate that first, intratubular amyloid is not a rare finding in kidney biopsies of patients with light chain cast nephropathy, and, second, it reflects an amyloidogenic capacity of light chains that can manifest as systemic light chain amyloidosis. Thus, intratubular amyloid should be systematically screened for in kidney biopsies from patients with light chain cast nephropathy and, if detected, should prompt a work-up for associated systemic light chain amyloidosis.

Modern Pathology (2018) 31, 452-462; doi:10.1038/modpathol.2017.124; published online 20 October 2017

Light chain cast nephropathy consists of the precipitation of monoclonal immunoglobulin light chains in the lumen of distal tubules, and is the most common paraprotein-associated pattern of kidney injury found during the course of multiple myeloma. ${ }^{1}$ Much more rarely, light chain cast nephropathy may occur with other monoclonal gammopathies, such as

Correspondence: Dr J-B Gibier, MD, Department of Pathology, Centre de Biologie Pathologie, CHU Lille, University of Lille, Lille F-59000, France.

E-mail: jeanbaptiste.gibier@chru-lille.fr

Received 27 May 2017; revised 2 August 2017; accepted 3 August 2017; published online 20 October 2017
Waldenström macroglobulinemia. ${ }^{2}$ Systemic light chain amyloidosis is another well-known complication of multiple myeloma, defined by the deposition of Congo red-positive, fibrillary monoclonal light chain complexes in renal parenchyma, as well as other vital organs, especially the heart. Coexistent light chain amyloid deposits are identified either at presentation or during the course of the disease in approximately $10-15 \%$ of multiple myeloma patients. ${ }^{3}$ Importantly, the occurrence of systemic light chain amyloidosis in multiple myeloma patients has been shown to be an adverse prognostic factor. ${ }^{4}$

The observation that the tubular casts of light chain cast nephropathy may show staining and 
ultrastructural characteristics of amyloid is a feature that has long been recognized., ${ }^{5,6}$ Purely intratubular amyloid is viewed as an unusual variant of light chain cast nephropathy with no clinical significance and, accordingly, 'does not merit the diagnosis of renal amyloidosis since the amyloid is exclusively intratubular and not deposited in the renal parenchyma'. ${ }^{7}$ However, the literature on this point only relies upon case reports ${ }^{5,8-13}$ and old clinicopathological studies, ${ }^{6,14-19}$ which did not properly assess the clinical significance of intratubular amyloid, especially its relationship with the occurrence of systemic light chain amyloidosis in extrarenal sites. Thus, we designed this retrospective study to evaluate the frequency of intratubular amyloid in light chain cast nephropathy biopsy specimens seen at a single academic institution, and to assess whether intratubular amyloid was associated with extrarenal systemic light chain amyloidosis.

\section{Materials and methods}

\section{Patient Selection}

Computerized records of the Department of Pathology, Lille University Hospitals, were searched to identify all kidney biopsy specimens with light chain cast nephropathy from 1 January 2002 to 31 December 2012. Clinical data, including hematological disease, gammopathy characteristics, renal presentation, serum creatinine and urinalysis (proteinuria, hematuria), treatment, and outcome data after kidney biopsy were obtained from patient electronic medical records. This study was done in accordance with the Declaration of Helsinki and was approved by our institutional review board. The referring physician obtained informed consent from each patient for use of the biopsy data, clinical data and leftover histological material for research. Acute kidney injury was classified using the Acute Kidney Injury Network (AKIN) criteria. Renal response was defined by an estimated glomerular filtration rate $\geq 30 \mathrm{ml} / \mathrm{min} / 1.73 \mathrm{~m}^{2}$ and/or dialysis independence at 3 months after biopsy. ${ }^{20}$

\section{Kidney Biopsies}

Two biopsy specimens were obtained from each patient: one was used for light microscopy and the other was snap-frozen for immunofluorescence; occasionally, the ends from one of the cores were taken for electron microscopy. Kidney biopsy samples for light microscopy were fixed in alcoholformalin-acetic acid, embedded in paraffin, and stained with Masson's trichrome, Jones methenamine silver, periodic acid-Schiff, hematoxylineosin-saffron and Congo red. The direct immunofluorescence assays were performed on $3-\mu \mathrm{m}$ cryostat sections according to standard procedures with anti- $\kappa$ (F0198; Dako), anti- $\lambda$ (F0199; Dako), anti-IgA
(F0204; Dako), anti-IgM (F0203; Dako), anti-IgG (F0202; Dako), anti-C3 (F0201; Dako), anti-C1q (F0254; Dako) and anti-fibrinogen (F0111; Dako) fluorescein isothiocyanate-conjugated antibodies. For electron microscopy, kidney tissue specimens were fixed in Carson fixative and postfixed in $1 \%$ osmium tetroxide, dehydrated with acetone, and embedded in Epon 812. The ultrathin sections were contrasted with uranylacetate and lead citrate, and studied using a LEO EM 906 electron microscope (Zeiss, Oberkochen, Germany).

All slides were reviewed by two pathologists. Light chain cast nephropathy was defined by the presence of typical fractured and polychromatophilic casts by light microscopy with staining by anti- $\kappa$ or anti- $\lambda$ conjugate by immunofluorescence. The number of casts was counted in 10 consecutive microscope fields at a magnification of $\times 200$. Interstitial fibrosis and tubular atrophy were graded on a semiquantitative scale, based on an estimate of the percentage of the renal cortex affected: $1-25 \%$ (mild), 26-50\% (moderate) or $>50 \%$ (severe). The diagnosis of intratubular amyloid was based on the visualization of intratubular casts showing typical apple-green birefringence in polarized light after Congo red staining, either on formalin or frozen sections. Congo red sections were also systematically examined under fluorescent light, which is more sensitive than light microscopy for the detection amyloid. ${ }^{21}$ The finding of amyloid, within renal parenchyma (glomeruli, vessels and/or interstitium) was an exclusion criterion.

\section{Extrarenal Amyloid Detection}

For each patient, all available histological material of extrarenal origin was systematically reviewed, with special attention to Congo red staining. When amyloid was detected, immunohistochemical typing on formalin-fixed and paraffin-embedded tissue was performed using anti-א (A0192; Dako), anti- $\lambda$ (A0193; Dako), anti-SAA (MC1; Dako) and anti-transthyretin (A0002; Dako) antibodies. All immunohistochemical studies were performed according to a standard automated immunohistochemical procedure (Ventana XT autostainer; Ventana Medical Systems ${ }^{\circledR}$, Benchmark XT, Strasbourg, France).

\section{Statistical Analysis}

Statistical analyses were performed using SAS $^{\circledR}$ software (SAS 9.4, SAS Institute Inc., Cary, NC, USA). Continuous variables are expressed as the means \pm s.d. or medians (interquartile ranges), as appropriate. Categorical variables are presented as absolute numbers and percentages. Comparisons between patients presenting with or without intratubular amyloid were made using chi-square or Fisher's tests for categorical variables, according to theoretical group numbers. The Student's $t$-test was 
used for continuous variable comparisons, after verification of normal distribution and variance equality by Shapiro \& Wilk's and Levene's tests. Predictive factors of renal response were identified by means of logistic models (PROC LOGISTIC in SAS $^{\circledR}$ ), using Firth's penalized maximum likelihood estimator for small sample sets. Continuous variables were tested for linearity and were dichotomized in case of violation. Multivariate models were built by including all covariates that were associated in univariate analyses, using a threshold of $P<0.10$ for selection, and suppressing redundant covariates. Interactions were systematically tested. Collinearity issues were assessed using the variance inflation factor calculation. The median overall survival and median follow-up times were estimated using Kaplan-Meier and inverse Kaplan-Meier methods, respectively (PROC LIFETEST). Univariate followed by multivariable Cox analyses were performed to identify independent predictors of survival (PROC PHREG). The log-linearity assumption for continuous variables and the proportional hazard assumption were tested by supremum tests. Multivariate Cox model types were built by including all covariates that were associated in univariate analyses, using a threshold of $P<0.10$ for selection, and suppressing redundant covariates. Interactions were systematically tested. All tests were two sided, and the criterion for statistical significance was a $P$-value of $<0.05$.

\section{Results}

\section{Patient Baseline Characteristics}

We analyzed 3126 consecutive native kidney biopsies performed in our center between 1 January 2002 and 31 December 2012. Sixty-six cases consistent with light chain cast nephropathy were eligible. In accordance with the exclusion criteria, four patients were excluded because of amyloid detected in the renal biopsy specimen (in glomeruli $(n=1)$, renal vessels $(n=2)$ or perirenal fat $(n=1))$. Two other patients were excluded because the pathological findings were not convincing and the clinical records ruled out a hematological disease. Therefore, 60 patients were included in this study. The clinical and biological characteristics of the patients at the time of the renal biopsy are summarized in Table 1. The cohort included 33 males (55\%) and 27 (45\%) females. The mean age was 66 years (range, 38-87). The underlying hematological disease was multiple myeloma $(n=56)$, Waldenström macroglobulinemia $(n=2)$ or mucosa-associated lymphoid tissue (MALT) lymphoma $(n=2)$. One of the light chain cast nephropathy case associated with Waldenström macroglobulinemia has been previously reported. ${ }^{22}$ Monoclonal light chains were of the $\lambda$ isotype in 32 cases and the $\kappa$ isotype in 28. Acute renal failure was the most frequent biopsy indication $(n=54)$,
Table 1 Baseline clinical and biological characteristics

\begin{tabular}{|c|c|}
\hline & Total $(\mathrm{n}=60)$ \\
\hline Age (range) & $66(38-87)$ \\
\hline $\operatorname{Sex}(F / M)$ & $27 / 33$ \\
\hline \multicolumn{2}{|l|}{ Precipitating factors } \\
\hline \# Hypercalcemia & 10 \\
\hline \# Drugs & 9 \\
\hline \# Sepsis/dehydration & 4 \\
\hline Acute kidney injury network stage $3(\%)$ & $41(68 \%)$ \\
\hline Serum creatinine $(\mathrm{mg} / \mathrm{dl})$ & $5.2 \pm 2.4$ \\
\hline $\begin{array}{l}\text { MDRD estimated glomerular filtration rate } \\
\left(\mathrm{ml} / \mathrm{min} / 1.73 \mathrm{~m}^{2}\right)\end{array}$ & $10.8(7.8-17.6)$ \\
\hline Hemodialysis required $^{\mathrm{a}}$ & $18(30 \%)$ \\
\hline Albuminemia (g/l) & $34.1 \pm 6.7$ \\
\hline Proteinuria (g/day) $(n=54)$ & $2.56(1.5-4.4)$ \\
\hline Bence-Jones proteinuria & $60(100 \%)$ \\
\hline Hematuria & $16(27 \%)$ \\
\hline \multicolumn{2}{|l|}{ Hematological disease } \\
\hline \# Multiple myeloma & 56 \\
\hline \# Waldenström macroglobulinemia & 2 \\
\hline \# Non-Hodgkin lymphoma & 2 \\
\hline $\begin{array}{l}\% \text { Plasma cell bone marrow infiltration } \\
(n=56)\end{array}$ & $32(18-49)$ \\
\hline$\beta 2$-microglobulin level (mg/l) $(\mathrm{n}=43)$ & $14.2 \pm 8.4$ \\
\hline Serum monoclonal protein (g/dl) & $1.3 \pm 1.6$ \\
\hline Heavy chain isotype (no/IgG/IgA/other) & $21 / 16 / 18 / 5$ \\
\hline Light chain isotype $(\kappa / \lambda)$ & $28 / 32$ \\
\hline Free light chain level (mg/dl) & $371(131-752)$ \\
\hline
\end{tabular}

${ }^{\mathrm{a}}$ Within 1 month after the kidney biopsy.

Table 2 Pathological characteristics

\# Glomeruli

Mean \% sclerotic glomeruli

\# Casts

Interstitial fibrosis/tubular atrophy

Mild

Moderate

Severe

Associated pathology

Light chain deposition disease

Light chain proximal tubulopathy

$13.5(10-20)$

$17 \pm 20$

$53.0(28-76)$

$19(32 \%)$

$28(47 \%)$

$13(22 \%)$

$7(\kappa: n=7)$

$2(\lambda: n=2)$

followed by progressive renal failure $(n=4)$ and isolated proteinuria $(n=2)$. A potential precipitating factor (hypercalcemia, drugs and dehydration/sepsis) was identified in 23 patients (Table 1).

The pathological features of light chain cast nephropathy are summarized in Table 2 and illustrated in Figure 1. A median of 53 (95\% CI 28-76) light chain casts were observed in 10 fields at a magnification of $\times 200$ by light microscopy. By immunofluorescence, the casts invariably matched the serum light chain isotype $(\lambda: n=32, \kappa: n=28)$. Interstitial fibrosis was graded as mild in 19 (32\%), moderate in $28(47 \%)$ and severe in $13(22 \%)$ cases. Seven patients had concomitant light chain deposition disease, characterized by linear light chain deposits by immunofluorescence ( $\kappa: n=7)$ and amorphous deposits by electron microscopy. Two patients had light chain proximal 

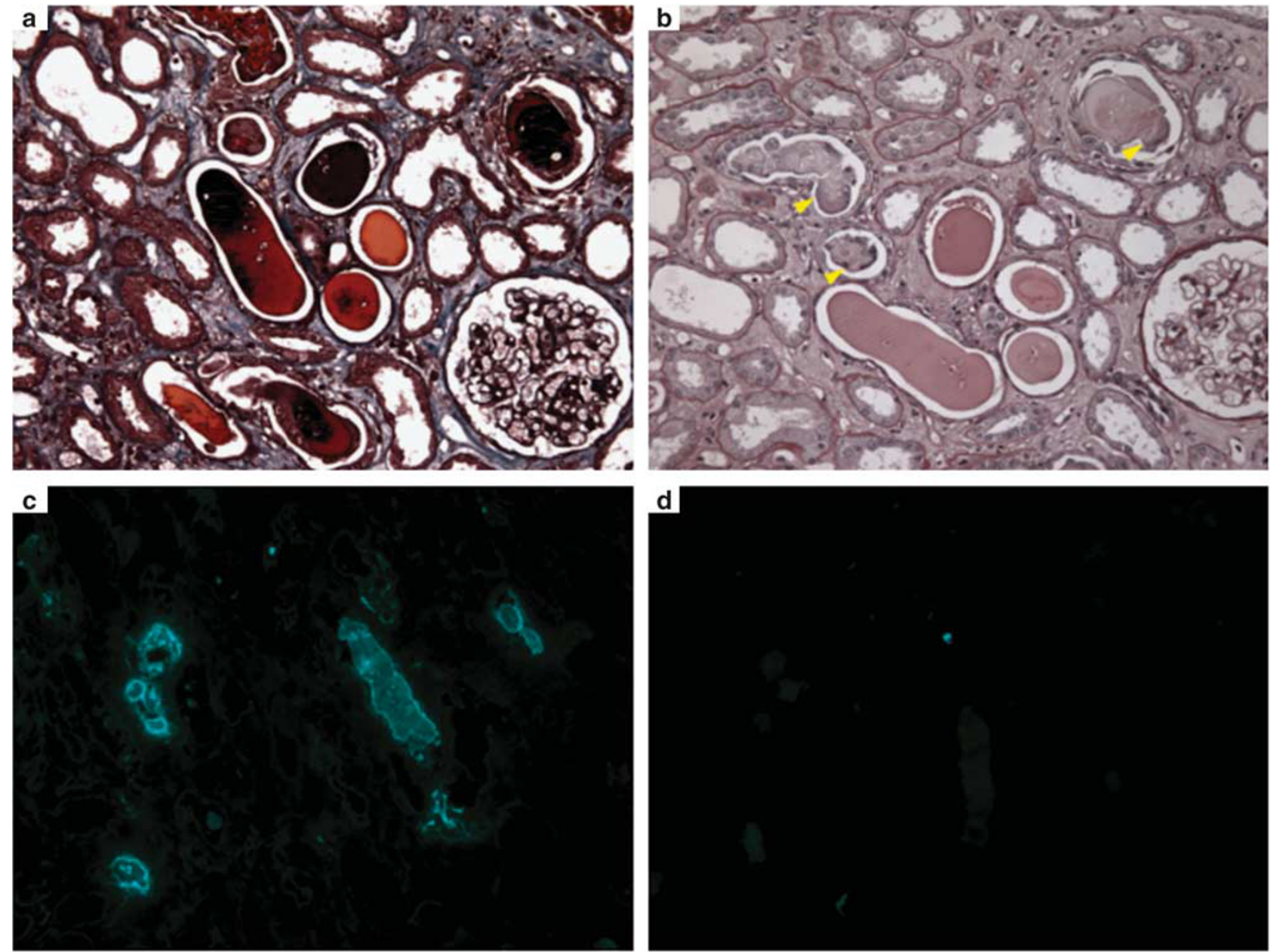

Figure 1 Light chain cast nephropathy histopathology. (a) Dense and fractured casts in the lumen of distal tubules (Masson trichrome, original magnification $\times 200$ ). (b) Weak staining of casts by periodic acid-Schiff with surrounding multinucleated giant cells (arrowheads) (periodic acid-Schiff, original magnification $\times 200$ ). (c) Staining of the casts with anti-k antibody (immunofluorescence $\times 200$ ). $(\mathbf{d})$ No staining with anti- $\lambda$ antibody (immunofluorescence $\times 200$ ).

tubulopathy $(\lambda: n=2)$ with intracytoplasmic crystals by electron microscopy.

\section{Intratubular Amyloid}

We found intratubular amyloid in 17 (28\%) of the 60 light chain cast nephropathy cases after systematic examination of Congo red-stained slides by light microscopy, polarized light and fluorescence (Figure 2). By definition, all 17 cases had at least one cast showing amyloid features (Figures 2a-d). The ratio of Congo red-positive/total casts was $<5 \%, 5-25 \%$, and $>25 \%$ for nine, three and five cases, respectively. We observed a 'rimmed' appearance of intratubular amyloid, that is, with more Congo red staining at the periphery of the cast, in 11/17 (65\%) cases (Figures 2e-l). Ultrastructural analysis of amyloid casts was performed in one case showing a rimmed pattern at light microscopy and revealed the presence of numerous non-branching fibrils randomly arrayed at the periphery of the cast
(Figures $2 \mathrm{~m}$ and $\mathrm{n}$ ). Amyloid was also detected as intracytoplasmic vacuoles in tubular cells in 4/17 cases (Figures 2o and p). These four cases did not show associated light chain proximal tubulopathy.

The clinico-biological characteristics at the time of the renal biopsy of the 'intratubular amyloid' group and the 'no intratubular amyloid' group are shown in Table 3. There was no statistical difference between the two groups for the mean level of serum creatinine, as well as other nephrological parameters. Intratubular amyloid was associated with the $\lambda$ light chain isotype (13/17 with $\lambda$ isotype in the 'intratubular amyloid' group vs 19/43 in the 'no intratubular amyloid' group, $P=0.02$ ).

\section{Extrarenal Systemic Light Chain Amyloidosis and Correlation with Intratubular Amyloid}

Extrarenal pathological specimens were available for 43 patients $(72 \%)$. They consisted mostly of bone marrow trephine biopsies, minor salivary gland 

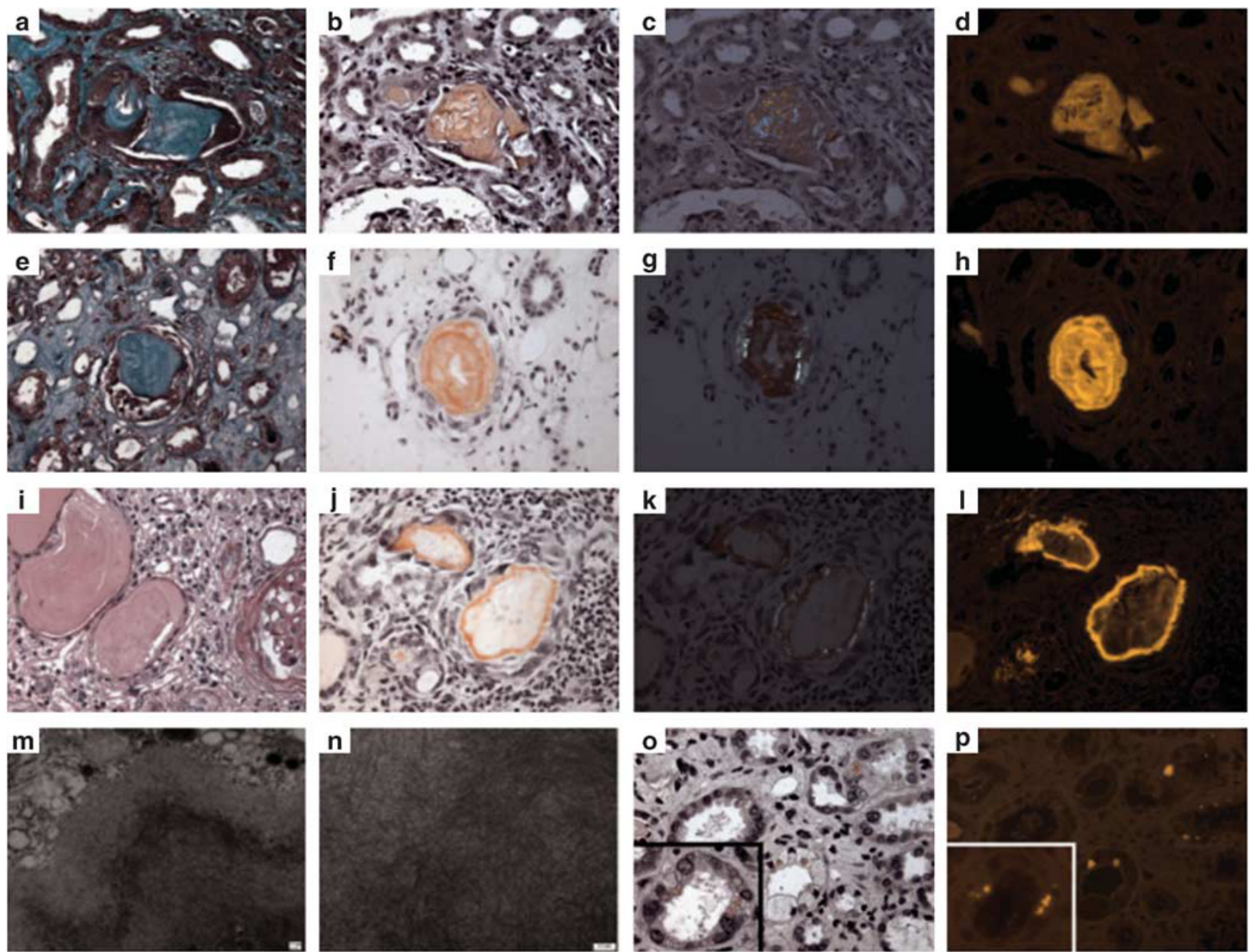

Figure 2 Examples of intratubular amyloid. Case 1: (a) intratubular casts (Masson trichrome, original magnification $\times 400$ ), (b) homogeneously stained by Congo red (Congo red under nonpolarized light, original magnification $\times 400$ ), (c) apple-green birefringence (Congo red under polarized light, original magnification $\times 400$ ) and (d) Congo red fluorescence (Congo red under fluorescence, original magnification $\times 400$ ). Case 2: (e) intratubular casts (Masson trichrome, original magnification $\times 400)$, $(\mathbf{f})$ plurilamellar rimmed pattern stained by Congo red (Congo red under nonpolarized light, original magnification $\times 400$ ), (g) apple-green birefringence (Congo red under polarized light, original magnification $\times 400)$ and $(\mathbf{h})$ Congo red fluorescence (Congo red under fluorescence, original magnification $\times 400$ ). Case 3: (i) intratubular casts (periodic acid-Schiff, original magnification $\times 400$ ), (j) rimmed pattern stained by Congo red (Congo red under nonpolarized light, original magnification $\times 400)$, $(\mathbf{k})$ apple-green birefringence (Congo red under polarized light, original magnification $\times 400$ ), (l) Congo red fluorescence (Congo red under fluorescence, original magnification $\times 400$ ) and fibrillar organization of amyloid at the periphery of the cast (electron microscopy, original magnification $\times 10000(\mathbf{m})$ and $\times 27800$ (n)). Amyloid within the cytoplasm of epithelial tubular cells stained with Congo red (Congo red under nonpolarized light, original magnification $\times 400$ (o) and Congo red fluorescence, original magnification $\times 400(\mathbf{p}))$.

biopsies and digestive tract biopsies and/or surgical specimens (Table 4). The number and the type of pathological specimens available did not differ between the 'intratubular amyloid' group and the 'no intratubular amyloid' group (13/17 patients with at least one extrarenal specimen in the 'intratubular amyloid' group vs 30/43 in the 'no intratubular amyloid' group, $P=0.6$ ). Moreover, the median period separating kidney biopsy from the collection of extrarenal samples did not differ between the two groups.

Congo red staining and immunohistochemistry revealed light chain amyloid in five ( $\lambda$ in four cases and $\kappa$ in one case) specimens corresponding to five patients. All five patients had intratubular amyloid on the renal biopsy. Conversely, no patient in the 'no intratubular amyloid' group was found to have extrarenal light chain amyloid, that is, intratubular amyloid correlated significantly with the occurrence of extrarenal light chain amyloid (5/13 with extrarenal light chain amyloid in the 'intratubular amyloid' group vs $0 / 30$ in the 'no intratubular amyloid' group, $P=0.001$ ) (Figure 3). In logistic regression analysis, intratubular amyloid was the only predictive factor for the occurrence of systemic light chain amyloidosis (odds ratio (OR) 38; 95\% CI $2-771, P=0.02$ ). We found extrarenal amyloid in minor salivary gland $(n=2)$, digestive tract $(n=1)$, 
Table 3 Comparison of the intratubular amyloid group and the no intratubular amyloid group

\begin{tabular}{|c|c|c|c|}
\hline & Intratubular amyloid $(\mathrm{n}=17)$ & $\begin{array}{l}\text { No intratubular amyloid } \\
\qquad(\mathrm{n}=43)\end{array}$ & $\mathrm{P}$-value \\
\hline Mean age (range) & $67(47-87)$ & $66(38-85)$ & 0.6 \\
\hline $\operatorname{Sex}(F / M)$ & $7 / 10$ & $20 / 23$ & 1 \\
\hline \multicolumn{4}{|l|}{ Precipitating factors } \\
\hline \# Hypercalcemia & 2 & 8 & 0.7 \\
\hline \# Drugs & 3 & 6 & 0.7 \\
\hline \# Sepsis/dehydration & 1 & 3 & 1 \\
\hline Acute kidney injury network stage $3(\%)$ & $11(65 \%)$ & $30(70 \%)$ & 0.7 \\
\hline Serum creatinine $(\mathrm{mg} / \mathrm{dl})$ & $5.4 \pm 2.7$ & $5.2 \pm 2.3$ & 0.7 \\
\hline $\begin{array}{l}\text { MDRD estimated glomerular filtration rate } \\
\left(\mathrm{ml} / \mathrm{min} / 1.73 \mathrm{~m}^{2}\right)\end{array}$ & $11.1(7.6-17.5)$ & $10.3(7.9-17.6)$ & 0.9 \\
\hline Hemodialysis required & $4(24 \%)$ & $14(33 \%)$ & 1 \\
\hline Albuminemia $(\mathrm{g} / \mathrm{l})$ & $34.1 \pm 6.6$ & $34.2 \pm 6.8$ & 1 \\
\hline Proteinuria (g/day) & $2.50(1.4-3.8)$ & $2.77(1.7-4.5)$ & 0.2 \\
\hline Hematuria & $6(35 \%)$ & $10(23 \%)$ & 0.3 \\
\hline \multicolumn{4}{|l|}{ Hematological disease } \\
\hline \# Multiple myeloma & 16 & 40 & 1 \\
\hline \# Waldenström macroglobulinemia & 1 & 1 & 1 \\
\hline \# Non-Hodgkin lymphoma & 0 & 2 & 1 \\
\hline$\%$ Plasma cell bone marrow infiltration $(n=56)$ & $35(24-60)$ & $30(15-42)$ & 0.4 \\
\hline$\beta 2$-microglobulin level (mg/l) & $14.2 \pm 9.1$ & $14.3 \pm 6.6$ & 1 \\
\hline Serum monoclonal protein (g/dl) & $1.2 \pm 1.8$ & $1.4 \pm 1.5$ & 0.2 \\
\hline Heavy chain isotype (no/IgG/IgA/other) & $5 / 3 / 8 / 1$ & $16 / 13 / 10 / 4$ & $0.8 / 0.5 / 0.1 / 1$ \\
\hline Light chain isotype $(\kappa / \lambda)$ & $4 / 13$ & $24 / 19$ & 0.02 \\
\hline Free light chain level (mg/dl) & $361(217.3-541.4)$ & $406(50-791.1)$ & 0.9 \\
\hline
\end{tabular}

Table 4 Extrarenal pathological specimens assessed for amyloid light chain amyloidosis

\begin{tabular}{|c|c|c|c|}
\hline & Intratubular amyloid & No intratubular amyloid & P-value \\
\hline \multicolumn{4}{|l|}{ Pathological specimens } \\
\hline Bone marrow biopsies $(n)$ & 4 & 15 & 0.5 \\
\hline Minor salivary gland biopsies & 5 & 11 & 0.7 \\
\hline Digestive tract biopsies/surgical specimens & 7 & 13 & 0.3 \\
\hline Other & 6 & 11 & 0.5 \\
\hline Patients with $\geq 1$ specimen & $13 / 17$ & $30 / 43$ & 0.6 \\
\hline \multicolumn{4}{|c|}{ Time between procurement of extrarenal specimen and renal biopsy, months (median, ranges) } \\
\hline Before kidney biopsy & $9(0-91)(n=11)$ & $14(0-191)(n=20)$ & 0.9 \\
\hline After kidney biopsy & $11(0-52)(n=10)$ & $8(0-60)(n=25)$ & 0.9 \\
\hline Amyloid light chain amyloidosis & $5 / 13$ & $0 / 30$ & 0.001 \\
\hline
\end{tabular}

bone marrow $(n=1)$ and muscle biopsies $(n=1)$. Extrarenal specimens containing amyloid were obtained after a mean period of 294 days (range +9 ; +607 ) after the diagnosis of light chain cast nephropathy on kidney biopsy.

\section{Treatments and Outcome}

Follow-up data were available for 59 of the 60 patients. The median duration of follow-up was 64 months (95\% CI 57-120) from the kidney biopsy. During the follow-up period, $78 \%$ of patients died. The median overall survival calculated from the time of kidney biopsy was 21 months (95\% CI
15-38). Light chain cast nephropathy was inaugural in 43 patients $(72 \%)$. There was no difference in the hematological treatment received between the 'intratubular amyloid' and 'no intratubular amyloid' groups. In particular, bortezomib and immunomodulatory drug (IMiD)-based (lenalidomide or thalidomide) therapies were equally distributed between the two groups (Table 5). A hematological response (at least partial response) was achieved in 40/59 $(68 \%)$ patients and was similar for the two groups. On multivariate analysis, obtainment of renal or hematological responses and inaugural light chain cast nephropathy significantly correlated with overall survival, whereas the presence or absence of intratubular amyloid did not (hazard ratio (HR) 0.5; 95\% CI 0.3-1.1; $P=0.1$ ) (Table 5). 

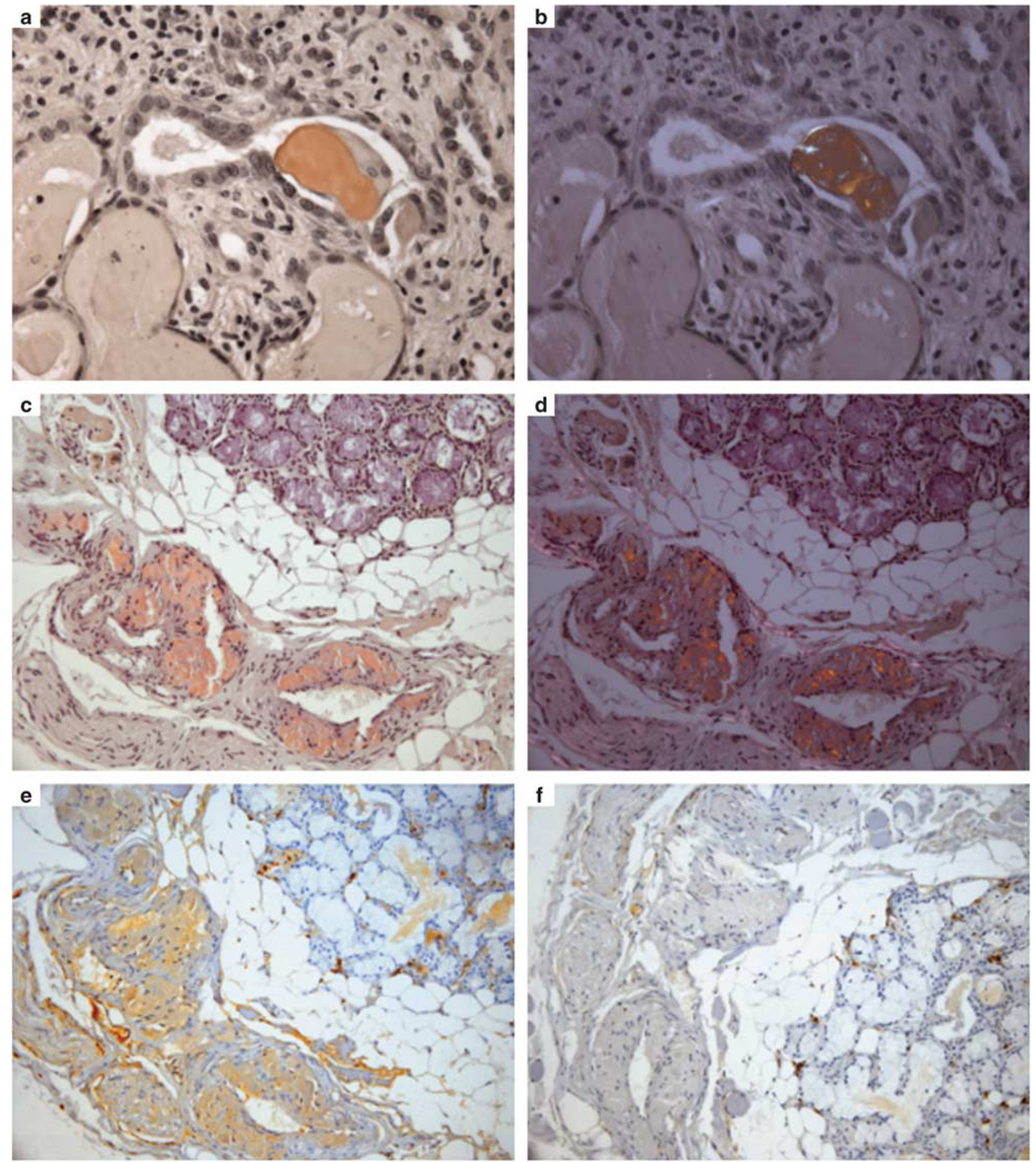

Figure 3 Example of an association between intratubular amyloid on kidney biopsy and systemic light chain amyloidosis. (a) intratubular amyloid (Congo red under nonpolarized light, original magnification $\times 400$ ) with $(\mathbf{b})$ apple-green birefringence (Congo red under polarized light, original magnification $\times 400$ ). (c) Minor salivary gland biopsy showing amyloid within vessels walls (Congo red under nonpolarized light, original magnification $\times 200$ ) with $(\mathbf{d})$ apple-green birefringence (Congo red under polarized light, original magnification $\times 200$ ), (e) amyloid staining with anti- $\lambda$ antibody (anti- $\lambda$ immunohistochemistry, original magnification $\times 200$ ) and (f) no staining with anti- $\kappa$ antibody (anti-k immunohistochemistry, original magnification $\times 200$ ).

Renal response was achieved in 19/59 (32\%) patients (Table 5). Among the clinical data, baseline estimated glomerular filtration rate, acute kidney injury network stage 3 , initial requirement for hemodialysis (within one month after the kidney biopsy) and $\lambda$ isotype were predictive of renal response on univariate analysis (Supplementary Table S1). The number of casts $(>30$ or $<30)$ and 
Table 5 Treatments and overall survival

\begin{tabular}{|c|c|c|c|c|}
\hline Characteristics & Total $(\mathrm{n}=59)$ & Intratubular amyloid $(\mathrm{n}=17)$ & No Intratubular amyloid $(\mathrm{n}=42)$ & P-value \\
\hline Bortezomib based $(n)$ & 41 & 11 & 30 & 0.8 \\
\hline IMiD based & 30 & 9 & 21 & 0.9 \\
\hline Both bortezomib and IMiD & 23 & 6 & 17 & 0.8 \\
\hline Neither & 11 & 3 & 8 & 1 \\
\hline Autologous stem cell transplant & 14 & 3 & 11 & 0.7 \\
\hline Hematological response & 40 & 11 & 29 & 0.8 \\
\hline Renal response $^{\mathrm{a}}$ & 19 & 6 & 13 & 0.8 \\
\hline Inaugural light chain cast nephropathy & 43 & 14 & 29 & 0.4 \\
\hline
\end{tabular}

\begin{tabular}{|c|c|c|c|}
\hline Prognostic indicators of overall survival (multivariate analysis) & Hazard ratio & 95\% Confidence interval & P-value \\
\hline Hematological response & 0.4 & $(0.2-0.8)$ & 0.005 \\
\hline Renal response $^{\mathrm{a}}$ & 0.4 & $(0.2-0.9)$ & 0.02 \\
\hline Presence of intratubular amyloid & 0.5 & $(0.3-1.1)$ & 0.1 \\
\hline Inaugural light chain cast nephropathy & 0.4 & $(0.2-0.8)$ & 0.005 \\
\hline
\end{tabular}

a Defined by estimated glomerular filtration rate $\geq 30 \mathrm{ml} / \mathrm{min} / 1.73 \mathrm{~m}^{2}$ and/or independence from dialysis at 3 months.

percentage of sclerotic glomeruli ( $>10 \%$ or $<10 \%$ ) were associated with renal response, whereas the presence or absence of intratubular amyloid was not (OR 1.2; 95\% CI 0.4-4.0, $P=0.7$ ). On multivariate analysis, only the requirement for hemodialysis at diagnosis and $\lambda$ isotype remained predictive (Supplementary Table S1).

\section{Discussion}

We undertook this investigation to determine the frequency of intratubular amyloid in 60 light chain cast nephropathy biopsy specimens, with the aim of correlating intratubular amyloid with the existence of extrarenal light chain amyloid. The primary result of our study is the finding of a significant association between intratubular amyloid and systemic light chain amyloidosis.

Intratubular amyloid was not rare in our series as we found Congo red-stained casts in $28 \%$ (17/60) of biopsy specimens with light chain cast nephropathy, regardless of their abundance. This prevalence is slightly less than in the three previously published clinicopathological studies of light chain cast nephropathy containing $>10$ cases, which reported the characteristics of the casts after using amyloid stains: $39 \%(13 / 33)$ in the study by Hill et al, ${ }^{17} 43 \%$ $(15 / 35)$ in the study by Limas et al ${ }^{14}$ and $58 \%(33 / 57)$ in the study by Vassar et al. ${ }^{6}$ Thioflavin T staining, which is less specific than Congo red, was used in the latter study for amyloid detection (Table 6). More recent light chain cast nephropathy clinicopathologic studies paid no attention to the amyloid staining properties of casts, emphasizing that intratubular amyloid is not currently considered to be clinically significant. ${ }^{1,20}$ In our study, intratubular amyloid casts frequently $(11 / 17,65 \%)$ showed a peculiar rimmed morphology consisting of a concentric organization and peripheral Congo red staining.
Formerly reported intratubular amyloid cases also mentioned a similar morphology (Table 6).

The main result of our study is the finding of an association between intratubular amyloid and systemic light chain amyloidosis. Indeed, we found light chain amyloidosis in 5/13 cases in the 'intratubular amyloid' group, whereas no patient was found to have light chain amyloidosis in the 'no intratubular amyloid' group (5/13 vs $0 / 30, P=0.001)$. These results suggest that light chains that form amyloid in tubular lumens can also form systemic amyloid deposits within tissues. This is supported by the finding that we observed intratubular amyloid more frequently in cases with $\lambda$ rather than $\kappa$ light chains (13/17 with $\lambda$ isotype in the 'intratubular amyloid' group vs 19/43 in the 'no intratubular amyloid' group, $P=0.02$ ), in accordance with the well-known greater amyloidogenicity of $\lambda$ than $\kappa$ chains. ${ }^{23}$ In the literature (reviewed in Table 6), the autopsy study by Melato et $a l^{16}$ is, to our knowledge, the only one to have systematically looked for a relationship between intratubular amyloid and systemic light chain amyloidosis based on the analysis of extrarenal tissues; they found no statistical association between intratubular amyloid and systemic light chain amyloidosis. However, the study had important limitations, including the low number of intratubular amyloid cases $(n=4)$ and the fact that organs frequently targeted by light chain amyloidosis such as bone marrow, the digestive tract and salivary glands, were not analyzed.

Our study reports the association of intratubular amyloid with systemic light chain amyloidosis diagnosed in extrarenal specimens (digestive tract, salivary glands, bone marrow and muscle) without renal parenchymal light chain amyloidosis at the time of the kidney biopsy. Such an association of intratubular amyloid with extrarenal amyloid, but without renal parenchymal amyloid, has been reported in three case reports: one case with 
Table 6 Review of the literature of intratubular amyloid

\begin{tabular}{|c|c|c|c|c|c|}
\hline References & $\begin{array}{c}\mathrm{Nb} \text { of cases with intratubular } \\
\text { amyloid }^{\mathrm{a}}\end{array}$ & $\begin{array}{l}\text { Rimmed } \\
\text { pattern }\end{array}$ & Cytoplasmic amyloid & Ultrastructural amyloid & $\begin{array}{l}\text { Extrarenal } \\
\text { amyloid }\end{array}$ \\
\hline Azzopardi et $a l^{5}$ & 1 & Yes & ND & ND & No \\
\hline Azzopardi et al ${ }^{24}$ & 1 & Yes & ND & ND & Yes $^{b}$ \\
\hline Vassar et $a l^{6 \mathrm{c}}$ & $33(57)$ & Yes & Yes & ND & ND \\
\hline Friman et $a l^{8}$ & 1 & Yes & ND & ND & Yes $^{\mathrm{d}}$ \\
\hline Limas et $a l^{14}$ & $15(35)$ & Yes & $3 / 15$ & Yes & $\mathrm{No}^{\mathrm{e}}$ \\
\hline Defronzo et al ${ }^{15}$ & $4(8)$ & Yes & ND & ND & ND \\
\hline Melato et $a 1^{16}$ & 4 & Yes & ND & ND & No \\
\hline Hill et $a l^{17}$ & $13(33)$ & ND & ND & ND & ND \\
\hline Pirani et $a 1^{18}$ & ND & Yes & ND & No & ND \\
\hline Rota et al ${ }^{19}$ & $1(26)$ & ND & ND & ND & ND \\
\hline El-Zoghby et $a l^{9}$ & 1 & Yes & Yes & Yes & Yes $^{f}$ \\
\hline Nasr et al ${ }^{10}$ & 1 & Yes & ND & Yes & ND \\
\hline Sethi et $a l^{11}$ & 1 & Yes & No & Yes & $\mathrm{No}^{\mathrm{g}}$ \\
\hline Hemminger et $a l^{25}$ & $\mathrm{No}^{h}$ & NA & Yes & Yes & ND \\
\hline Larsen et $a l^{38}$ & $\mathrm{No}^{\mathrm{h}}$ & NA & Yes & Yes & ND \\
\hline Kato et $a l^{12}$ & 1 & Yes & No & Yes & ND \\
\hline Iliuta et al ${ }^{13}$ & 1 & No & Yes & Yes & ND \\
\hline Current study & $17(60)$ & Yes & Yes & Yes & $5 / 13$ \\
\hline
\end{tabular}

Abbreviations: NA, not applicable; ND, not determined.

a Number of cases with intratubular amyloid (total number of cases with light chain cast nephropathy).

${ }^{\mathrm{b}}$ Skin, flexor retinaculum and omental fat.

${ }^{\mathrm{C}}$ Use of thioflavin $\mathrm{T}$ for amyloid staining.

dSubcutaneous amyloid tumors.

e The presence of amyloid-containing tubular casts was apparently independent of the presence or extent of infiltration in other organs.

${ }^{\mathrm{f}}$ Bone marrow and synovia.

gNo amyloid in bone marrow.

heurely intracytoplasmic amyloid.

subcutaneous amyloid tumors ${ }^{8}$ one case with skin, joint and omental fat involvement, ${ }^{24}$ and one with bone marrow, joint and peripheral nerve involvement. ${ }^{9}$ This intriguing observation, in conjunction with the fact that systemic light chain amyloidosis was diagnosed, on average, 294 days after the renal biopsy, may indicate that intratubular amyloid is an early phenomenon during light chain cast nephropathy-associated amyloidosis and precedes the formation of light chain amyloid within tissues, either renal or extrarenal.

Although the exact pathophysiology of intratubular amyloid remains unclear, its possible association with amyloid found within the cytoplasm of tubular epithelial cells suggests that intratubular amyloid was formed intracytoplasmically from reabsorbed light chains and subsequently secreted into the lumen. ${ }^{25}$ This hypothesis is in agreement with studies suggesting that proteolytic enzyme processing of immunoglobulin light chains within lysosomes is central to amyloid fibril formation. ${ }^{26}$ Experimental models of renal light chain amyloidosis have shown that mesangial cells have a central role in amyloidogenesis through endocytosis and subsequent intra-lysosomal proteolysis of light chains. ${ }^{27,28}$ It is possible that a similar mechanism may occur in tubular epithelial cells and lead to the production of intratubular amyloid. The formation of amyloid in the urinary space, secondarily endocytosed by proximal tubular cells, is an alternative possibility. ${ }^{13}$
In this study, we found that Acute Kidney Injury Network stage 3, baseline estimated glomerular filtration rate and requirement for hemodialysis were prognostic factors of renal response, by univariate analysis, as reported in other studies. ${ }^{20,29}$ The good prognosis associated with $\lambda$ isotype is difficult to interpret in the absence of available urinary light chain excretion levels. ${ }^{30}$ The number of casts was also predictive of renal response; this pathological factor has been recently highlighted in a previous study. ${ }^{20}$ We could find no difference in the prognosis between light chain cast nephropathy patients with or without intratubular amyloid, either for renal response or overall survival. It is possible that potential prognostic differences related to the amyloidogenicity of light chains in intratubular amyloid patients could have been obscured by the generally bad outcome of light chain cast nephropathy. However, it should be noted that the renal prognosis of multiple myeloma patients is improving, ${ }^{31,32}$ likely due to more effective chemotherapy molecules, such as thalidomide and bortezomib, ${ }^{33}$ and the use of new dialysis techniques to remove free light chains. ${ }^{34}$

Our results suggest that the detection of intratubular amyloid could help to identify light chain cast nephropathy patients at risk of developing systemic light chain amyloidosis. This point is of importance, as the recognition of systemic light chain amyloidosis associated with multiple myeloma helps to determine prognosis and treatment. ${ }^{35,36}$ Indeed, the 
occurrence of systemic light chain amyloidosis in patients with multiple myeloma has been shown to be an adverse prognostic factor. ${ }^{4}$ Thus, we believe that renal biopsy specimens showing light chain cast nephropathy should be systematically screened for intratubular amyloid through the examination of casts after Congo red staining. Patients with intratubular amyloid should be considered to be at risk for the development of systemic light chain amyloidosis and, subsequently, a specific work-up and surveillance for light chain amyloidosis may be necessary for these patients. Finally, our results can be viewed as a supplementary justification to perform kidney biopsy in patients suspected to have light chain cast nephropathy. ${ }^{37}$

This study has important limitations inherent to its retrospective design. In particular, extrarenal specimens were available for the assessment of amyloid for only $72 \%$ of patients. However, our results suggest, for the first time, that intratubular amyloid is not a histological peculiarity with no clinical significance, but conversely reflects the amyloidogenic capacity of light chains that can manifest as systemic light chain amyloidosis. The routine pathological examination of casts with Congo red to identify intratubular amyloid should be mandatory in all patients with light chain cast nephropathy. From a clinical point of view, the detection of intratubular amyloid should trigger the search for systemic light chain amyloidosis.

\section{Acknowledgments}

We gratefully acknowledge the colleagues who participated in this study: YM Robin (Centre Oscar Lambret, Lille), P Gosset (Hôpital Saint Vincent, Lille), F Carpentier (CH Roubaix), C Fromentin (CH Lens), S Bisiau (CH Valenciennes), M Parent (Douai Pathologie), O Riviere (Centre de Pathologie, Saint Omer), T Malard (Centre de Pathologie, Valenciennes), PY Paquet (Liberté Pathologie, Lille), J Delplace (Nordpathologie, Lille), T Facon (CHU de Lille), B Dupriez (CH Lens), C Rose (Hôpital Saint Vincent) and M Wetterwald (Dunkerque). We thank RM Siminski and MH Gevaert (Histology Department, CHU de Lille), and all the technical staff of the Pathology Department of the CHU de Lille for their technical assistance.

\section{Disclosure/conflict of interest}

The authors declare no conflict of interest.

\section{References}

1 Nasr SH, Valeri AM, Sethi S, et al. Clinicopathologic correlations in multiple myeloma: a case series of 190 patients with kidney biopsies. Am J Kidney Dis 2012;59:786-794.
2 Vos JM, Gustine J, Rennke HG, et al. Renal disease related to Waldenstrom macroglobulinaemia: incidence, pathology and clinical outcomes. Br J Haematol 2016;175:623-630.

3 Rajkumar SV, Gertz MA, Kyle RA. Primary systemic amyloidosis with delayed progression to multiple myeloma. Cancer 1998;82:1501-1505.

4 Vela-Ojeda J, Garcia-Ruiz Esparza MA, PadillaGonzalez Y, et al. Multiple myeloma-associated amyloidosis is an independent high-risk prognostic factor. Ann Hematol 2009;88:59-66.

5 Azzopardi JG. Clinicopathologic Conference. A case of plasma cell leukemia. Br Med J 1962;1:169-177.

6 Vassar PS, Culling CF. Fluorescent amyloid staining of casts in myeloma nephrosis. Arch Pathol 1962;73: 59-63.

7 D’Agati VD, Jennette JC, Silva FG. Plasma cell dyscrasia: tubulointerstitial involvement. In: D'Agati VD, Jennette JC, Silva FG (eds). Non-Neoplastic Kidney Disease. Atlas of Nontumor Pathology, Fascicle 4. ARP Press: Washington, 2005, pp 603-623.

8 Friman C, Tornroth T, Wegelius O. IgD myeloma associated with multiple extramedullary amyloidcontaining tumours and amyloid casts in the renal tubules. Ann Clin Res 1970;2:161-166.

9 El-Zoghby Z, Lager D, Gregoire J, et al. Intra-tubular amyloidosis. Kidney Int 2007;72:1282-1288.

10 Nasr SH, Alobeid BB, Otrakji JA, et al. Myeloma cast nephropathy, direct renal infiltration by myeloma, and renal extramedullary hematopoiesis. Kidney Int 2008;73:517-518.

11 Sethi S, Hanna MH, Fervenza FC. Unusual casts in a case of multiple myeloma. Am J Kidney Dis 2009;54: 970-974.

12 Kato H, Fujigaki Y, Asakawa S, et al. Rapid deterioration of the renal function caused by the coexistence of intratubular amyloidosis and myeloma cast nephropathy. Intern Med 2015;54:3023-3028.

13 Iliuta I, Garneau AP, Latulippe E, et al. Amyloid cast tubulopathy: a unique form of immunoglobulininduced renal disease. Blood Cancer J 2016;6:e474.

14 Limas C, Wright JR, Matsuzaki M, et al. Amyloidosis and multiple myeloma. A reevaluation using a control population. Am J Med 1973;54:166-173.

15 Defronzo RA, Humphrey RL, Wright JR, et al. Acute renal failure in multiple myeloma. Medicine (Baltimore) 1975;54:209-223.

16 Melato M, Falconieri G, Pascali E, et al. Amyloid casts within renal tubules: a singular finding in myelomatosis. Virchows Arch A Pathol Anat Histol 1980;387:133-145.

17 Hill GS, Morel-Maroger L, Mery JP, et al. Renal lesions in multiple myeloma: their relationship to associated protein abnormalities. Am J Kidney Dis 1983;2:423-438.

18 Pirani CL, Silva F, D'Agati V, et al. Renal lesions in plasma cell dyscrasias: ultrastructural observations. Am J Kidney Dis 1987;10:208-221.

19 Rota S, Mougenot B, Baudouin B, et al. Multiple myeloma and severe renal failure: a clinicopathologic study of outcome and prognosis in 34 patients. Medicine (Baltimore) 1987;66:126-137.

20 Ecotiere L, Thierry A, Debiais-Delpech C, et al. Prognostic value of kidney biopsy in myeloma cast nephropathy: a retrospective study of 70 patients. Nephrol Dial Transplant 2016;31:64-72.

21 Linke RP. Highly sensitive diagnosis of amyloid and various amyloid syndromes using Congo red fluorescence. Virchows Arch 2000;436:439-448. 
22 Gnemmi V, Leleu X, Provot F, et al. Cast nephropathy and light-chain deposition disease in Waldenstrom macroglobulinemia. Am J Kidney Dis 2012;60: 487-491.

23 Solomon A WD. Protein host factors implicated in the pathogenesis of light chain amyloidosis (AL amyloidosis). Amyloid 1995;2:269-279.

24 Azzopardi JG, Lehner T. Systemic amyloidosis and malignant disease. J Clin Pathol 1966;19:539-548.

25 Hemminger J, Satoskar A, Brodsky SV, et al. Unique pattern of renal kappa light chain amyloid deposition with histiocytic transdifferentiation of tubular epithelial cells. Am J Surg Pathol 2012;36: 1253-1257.

26 Shirahama T, Cohen AS. Intralysosomal formation of amyloid fibrils. Am J Pathol 1975;81:101-116.

27 Keeling J, Teng J, Herrera GA. AL-amyloidosis and light-chain deposition disease light chains induce divergent phenotypic transformations of human mesangial cells. Lab Invest 2004;84:19.

28 Tagouri YM, Sanders PW, Picken MM, et al. In vitro AL-amyloid formation by rat and human mesangial cells. Lab Invest 1996;74:290-302.

29 Dimopoulos MA, Roussou M, Gkotzamanidou M, et al. The role of novel agents on the reversibility of renal impairment in newly diagnosed symptomatic patients with multiple myeloma. Leukemia 2013;27:423-429.

30 Drayson M, Begum G, Basu S, et al. Effects of paraprotein heavy and light chain types and free light chain load on survival in myeloma: an analysis of patients receiving conventional-dose chemotherapy in
Medical Research Council UK multiple myeloma trials. Blood 2006;108:2013-2019.

31 Reule S, Sexton DJ, Solid CA, et al. ESRD due to multiple myeloma in the United States, 2001-2010. J Am Soc Nephrol 2016;27:1487-1494.

32 Decourt A, Gondouin B, Delaroziere JC, et al. Trends in survival and renal recovery in patients with multiple myeloma or light-chain amyloidosis on chronic dialysis. Clin J Am Soc Nephrol 2016;11:431-441.

33 Dimopoulos MA, Delimpasi S, Katodritou E, et al. Significant improvement in the survival of patients with multiple myeloma presenting with severe renal impairment after the introduction of novel agents. Ann Oncol 2014;25:195-200.

34 Hutchison CA, Cockwell P, Reid S, et al. Efficient removal of immunoglobulin free light chains by hemodialysis for multiple myeloma: in vitro and in vivo studies. J Am Soc Nephrol 2007;18:886-895.

35 Bahlis NJ, Lazarus HM. Multiple myeloma-associated AL amyloidosis: is a distinctive therapeutic approach warranted? Bone Marrow Transplant 2006;38:7-15.

36 Madan S, Dispenzieri A, Lacy MQ, et al. Clinical features and treatment response of light chain (AL) amyloidosis diagnosed in patients with previous diagnosis of multiple myeloma. Mayo Clin Proc 2010;85:232-238.

37 Leung N. To biopsy or not to biopsy, that is the question in myeloma cast nephropathy. Nephrol Dial Transplant 2016;31:1-3.

38 Larsen CP, Borrelli GS, Walker PD. Amyloid proximal tubulopathy: a novel form of light chain proximal tubulopathy. Clin Kidney J 2012;5:130-132.

Supplementary Information accompanies the paper on Modern Pathology website (http://www.nature.com/ modpathol) 